OPEN ACCESS

Edited by: Hamid Mirzaei,

Tehran University of Medical Sciences, Iran

Reviewed by:

Sherly Mardiana

University of Pennsylvania,

United States

Brian Till,

Fred Hutchinson Cancer Research

Center, United States

*Correspondence: John Maher

john.maher@kcl.ac.uk

Specialty section: This article was submitted to Cancer Immunity and Immunotherapy, a section of the journal Frontiers in Immunology

Received: 15 December 2021 Accepted: 20 January 2022 Published: 09 February 2022

Citation:

Halim L, Das KK, Larcombe-Young D, Ajina A, Candelli A, Benjamin R, Dillon R, Davies DM and Maher J (2022) Engineering of an AvidityOptimized CD19-Specific Parallel

Chimeric Antigen Receptor

That Delivers Dual CD28 and 4-1BB Co-Stimulation.

Front. Immunol. 13:836549. doi: 10.3389/fimmu.2022.836549

\section{Engineering of an Avidity-Optimized CD19-Specific Parallel Chimeric Antigen Receptor That Delivers Dual CD28 and 4-1BB Co-Stimulation}

\author{
Leena Halim ${ }^{1}$, Kushal K. Das ${ }^{2}$, Daniel Larcombe-Young ${ }^{1}$, Adam Ajina ${ }^{1}$, Andrea Candelli ${ }^{2}$, \\ Reuben Benjamin ${ }^{3,4}$, Richard Dillon ${ }^{5,6}$, David M. Davies ${ }^{1}$ and John Maher ${ }^{1,7,8,9 *}$ \\ ${ }^{1}$ Chimeric Antigen Receptor (CAR) Mechanics Laboratory, Guy's Cancer Centre, School of Cancer and Pharmaceutical \\ Sciences, King's College London, London, United Kingdom, ${ }^{2}$ Lumicks, Amsterdam, Netherlands, ${ }^{3}$ Faculty of Life Sciences and \\ Medicine, School of Cancer and Pharmaceutical Sciences, King's College London, London, United Kingdom, ${ }^{4}$ Department of \\ Clinical Haematology, King's College Hospital National Health Service (NHS) Foundation Trust, London, United Kingdom, \\ ${ }^{5}$ Department of Clinical Haematology, Guy's and St Thomas' National Health Service (NHS) Foundation Trust, London, United \\ Kingdom, ${ }^{6}$ Department of Medicine and Molecular Genetics, King's College London, London, United Kingdom, ${ }^{7}$ Leucid Bio, \\ Guy's Hospital, London, United Kingdom, ${ }^{8}$ Department of Clinical Immunology and Allergy, King's College Hospital National \\ Health Service (NHS) Foundation Trust, London, United Kingdom, ${ }^{9}$ Department of Immunology, Eastbourne Hospital, \\ Eastbourne, United Kingdom
}

Co-stimulation is critical to the function of chimeric antigen receptor (CAR) T-cells. Previously, we demonstrated that dual co-stimulation can be effectively harnessed by a parallel (p)CAR architecture in which a CD28-containing second generation CAR is coexpressed with a 4-1BB containing chimeric co-stimulatory receptor (CCR). When compared to linear CARs, pCAR-engineered T-cells elicit superior anti-tumor activity in a range of pre-clinical models. Since CD19 is the best validated clinical target for cellular immunotherapy, we evaluated a panel of CD19-specific CAR and pCAR T-cells in this study. First, we generated a panel of single chain antibody fragments (scFvs) by alanine scanning mutagenesis of the CD19-specific FMC63 scFv ( $\mathrm{V}_{H}$ domain) and these were incorporated into second generation $\mathrm{CD} 28+\mathrm{CD} 3 \zeta$ CARs. The resulting panel of CAR Tcells demonstrated a broad range of CD19 binding ability and avidity for CD19-expressing tumor cells. Each scFv-modified CAR was then converted into a pCAR by co-expression of an FMC63 scFv-targeted CCR with a 4-1BB endodomain. When compared to second generation CARs that contained an unmodified or mutated FMC63 scFv, each pCAR demonstrated a significant enhancement of tumor re-stimulation potential and IL-2 release, reduced exhaustion marker expression and enhanced therapeutic efficacy in mice with established Nalm-6 leukemic xenografts. These data reinforce the evidence that the pCAR platform delivers enhanced anti-tumor activity through effective provision of dual co-stimulation. Greatest anti-tumor activity was noted for intermediate avidity CAR T-cells and derived pCARs, raising the possibility that effector to target cell avidity is an important determinant of efficacy.

Keywords: chimeric antigen receptor, avidity, CD19, parallel CAR, co-stimulation, CD28, 4-1BB 


\section{INTRODUCTION}

Chimeric antigen receptors (CARs) are fusion molecules that redirect lymphocyte specificity against cell surface targets. Immunotherapy with CD19-specific CAR T-cells has achieved dramatic impact in the treatment of relapsed/refractory B-cell leukemias and lymphomas. The key event that propelled this technology to become the largest growth area in immunooncology (1) was the inclusion of either CD28 or 4-1BB costimulatory elements within the CAR endodomain $(2,3)$. Evaluation of these so-called second generation (2G) CARs in human T-cells revealed that they confer a significantly enhanced capacity to mediate target-dependent proliferation and cytokine release, when compared to first generation (1G) receptors that only deliver an activating signal $(4,5)$. Currently, there are four licensed CD19-specific CAR T-cell products available worldwide, all of which contain an FMC63 single chain antibody fragment (scFv) and either a CD28 or 4-1BB co-stimulatory domain. Given the therapeutic failure of earlier designs, this highlights the vital importance of co-stimulation in this clinical breakthrough.

There is considerable evidence that combined provision of both CD28 and 4-1BB co-stimulation can synergistically enhance T-cell immune responses (6-8). Although these receptors activate overlapping signaling pathways, strength and kinetics of response differ markedly. While CD28-containing CARs elicit faster and larger scale signaling flux, 4-1BB favors a less intense but longer lasting response (9). We have previously shown that CAR T-cell co-stimulation both by CD28 and 4-1BB is optimally delivered by two separate fusion receptors. To leverage this, we engineered parallel (p)CARs in which a CD28-containing $2 \mathrm{G}$ CAR is co-expressed with a $4-1 \mathrm{BB}$ containing chimeric co-stimulatory receptor (CCR) (10). We observed that pCAR T-cells demonstrate more durable activity in several tumor models, indicated by enhanced proliferation, cytokine release and cytolytic function and lowered expression of exhaustion and senescence markers. As a result, superior in vivo anti-tumor activity was consistently achieved by pCAR Tcells compared to linear CAR T-cells in which either CD28 or 4$1 \mathrm{BB}(2 \mathrm{G}$ CAR) $(4,5)$ or both of these domains (third generation CAR) (11) had been included. Moreover, we also observed that pCAR T-cells outperformed the combination of a 1G CAR and a dual CD28 + 4-1BB CCR (12), potentially consistent with the importance of membrane proximity in effective co-stimulation. In mice in which pCAR T-cells achieved complete tumor rejection, enhanced functional persistence of these cells was confirmed by successful rejection of a delayed tumor rechallenge (10).

CD19 is the most strongly validated target antigen for CAR Tcell immunotherapy. Unprecedented efficacy has been reported by several groups using CD19-specific CAR T-cells to treat relapsed refractory B-cell malignancy (13). However, while CD19-specific CAR T-cells have proven clinically transformative, they are not uniformly successful in the induction of complete remission (CR) of B-cell malignancy. In lymphoma, $\mathrm{CR}$ rates range between 50 $67 \%$, of which $40-63 \%$ are durable to $12-29$ months. In B-cell ALL, the $\mathrm{CR}$ rate is higher at $69-92 \%$, but relapse occurs in $21-58 \%$ within 14 months (14). Moreover, greater than $50 \%$ of patients who receive CD19-specific CAR T-cell immunotherapy ultimately develop progressive disease (15). Relapse in which CD19 expression is maintained is the most common scenario particularly with CD28-containing 2G CARs - and this has been linked to limited persistence of CAR T-cells (16-18). Given the need for more potent and durable CAR T-cell strategies to target CD19, we set out here to develop CD19-targeted pCARs in order to test their potential utility in models of B-cell malignancy. To test the importance of relative avidity of the CAR and CCR targeting moiety within the PCAR, we generated a panel of CARs with differing relative avidity for $\mathrm{CD} 19$ and co-expressed these with a CCR in which an unmodified FMC63 scFv was used to confer target specificity.

\section{MATERIALS AND METHODS}

\section{Cell Lines}

Nalm-6 cells were a gift of Dr Robert Köchl, (the Francis Crick Institute, London, UK). LO68 were a gift from Prof T Sethi and Raji cells a gift from Dr Linda Barber (both King's College London, London, UK). 293T cells were obtained from the European Collection of Authenticated Cell Cultures. Tumor cell lines were grown in R10 or D10 medium, respectively comprising RPMI or DMEM supplemented with 10\% FBS and GlutaMax. 293VEC$\mathrm{RD} 114^{\mathrm{TM}}$ cells retroviral packaging cells were a gift of Dr Manuel Caruso (Biovec Pharma, Québec, Canada) and were maintained in D10. Cell lines were validated by STR typing and were routinely monitored for mycoplasma contamination.

\section{Human Samples}

Blood samples from healthy male and females aged between 1865 years were obtained from healthy volunteers with approval of a National Health Service Research Ethics Committee (reference 09/H0804/92 and 18/WS/0047).

\section{Retroviral Constructs}

All recombinant DNA constructs were expressed using the SFG retroviral vector. Complementary DNA encoding for the FMC63 $\mathrm{scFv}$ was designed using published sequences (19) (GenBank HM852952.1) and synthesized by Integrated DNA Technologies (Coralville, IA, USA). The second-generation F-2 CAR was designed as follows; a CD8 $\alpha$ leader was fused to the FMC63 scFv (arranged as variable light $\left(\mathrm{V}_{\mathrm{L}}\right)$ - variable heavy $\left(\mathrm{V}_{\mathrm{H}}\right)$ domains separated by a $[\text { SerGly } 4]_{3}$ linker). The synthetic cDNA was flanked with a $5^{\prime} \mathrm{Ncol}$ restriction site (that coincides with the start codon) and a 3' Not1 restriction site. This fragment was cloned into the unique Ncol and Not1 restriction sites of SFG A20-28z (20), placing the scFv cDNA upstream of the fused MYC epitope tag_CD28 hinge/ transmembrane/endodomain_CD3 $\zeta$ endodomain.

The complementarity determining region (CDR) 3 of the $\mathrm{V}_{\mathrm{H}}$ domain within the FMC63 scFv was identified using Abysis. ${ }^{1}$ To generate CAR variants with an altered ability to bind CD19, an alanine (A) residue was substituted for the first or second glycine

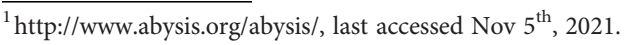


(G01, G02) or alternatively for the third, fourth or fifth tyrosine (Y03-Y05) within the CDR3 region of the $\mathrm{V}_{\mathrm{H}}$ domain. These substitutions were introduced into the F-2 CAR via single site mutagenesis (Genscript, Piscataway, NY, USA).

Codon optimized cDNAs encoding candidate pCARs were synthesized by Genscript (Piscataway, NJ, USA). To engineer pCARs, an Ncol flanked CCR cDNA was engineered that comprises a linear fusion of the following elements: a colonystimulating factor-1 receptor leader peptide, FMC63 scFv binding domain $\left(\mathrm{V}_{\mathrm{L}}-\mathrm{V}_{\mathrm{H}}\right.$ order), $\mathrm{CD} 8 \alpha$ spacer and transmembrane domain (codons 137-208), a 4-1BB co-stimulatory endodomain (codons 214-255), C-terminal FLAG epitope tag, an RRKR furin cleavage site, SGSG linker and Porcine Teschovirus (P2A) ribosomal skip peptide. This cDNA fragment was inserted into the unique Ncol restriction site containing the start codon of each the $\mathrm{V}_{\mathrm{H}}$ CDR3mutated CARs within SFG, thereby placing the CCR cDNA upstream of the CAR cDNA. Codon wobbling was used to minimize direct repeats within these vector inserts.

A codon optimized cDNA encoding for human CD19 was synthesized by Genscript and cloned into the Ncol site of SFG. The SFG ffLuc/RFP vector which encodes both firefly luciferase and dsTomato red fluorescent protein has been described previously (20).

\section{Transduction and Expansion of Human T-Cells}

Viral vector was prepared as described using 293VEC-RD $114^{\mathrm{TM}}$ cells (21) or by triple transfection of $293 \mathrm{~T}$ cells. In brief, $1.65 \times 10^{6}$ low passage $293 \mathrm{~T}$ cells in $11 \mathrm{~mL}$ IMDM $+10 \%$ FBS were evenly distributed in a $10 \mathrm{~cm}$ plate. After $8-24 \mathrm{~h}$, GeneJuice $(30 \mu \mathrm{L}$; Sigma-Aldrich, Poole UK; Cat\# 70967) was added to $470 \mu \mathrm{L}$ IMDM (no serum) and mixed gently. After incubation for 5 minutes at room temperature, $3.125 \mu \mathrm{g}$ RD114 plasmid (a gift of Prof M Collins, University College London, London UK), 4.6875 $\mu \mathrm{g}$ pEQ-Pam3 plasmid (a gift of Dr M Pulé, University College London, London UK) and $4.6875 \mu \mathrm{g}$ SFG vector of interest were added to the GeneJuice/medium mixture, mixed gently and incubated for 15 minutes at room temperature. The transfection mixture was dropwise to the plate and gently swirled to ensure even distribution. After incubation for $48 \mathrm{~h}$ at $37^{\circ} \mathrm{C}, 5 \%$ $\mathrm{CO}_{2}$, medium was removed for snap freezing using an ethanol dry ice bath and replaced. After a further $24 \mathrm{~h}$, this procedure was repeated. Frozen virus was stored in aliquots at $-80^{\circ} \mathrm{C}$. Retroviral transduction and culture of phytohemagglutinin- or CD3+CD28 Dynabead-activated T-cells using RetroNectin (Takara, Orchard Parkway, San Jose, CA, USA; Cat\# T100B)-coated plasticware was performed as described $(4,22)$.

\section{Flow Cytometry Analysis}

All cell surface antigen staining reactions were performed for 30 min on ice. CAR expression was detected with mouse 9e10 hybridoma supernatant $(20 \mu \mathrm{L}$ per test, produced in house) followed by goat anti-mouse IgG/A/M-RPE (Dako/Agilent, Santa Clara CA, USA; Cat\# R048001-2) or Alexa Fluor ${ }^{\circledR} 647$ goat anti-mouse/human IgG (Jackson ImmunoResearch, Europe Ltd, Ely, UK; Cat\# 115-605-003; RRID: AB_2338902). CD19 expression was detected using FITC anti-human CD19
(Biolegend, San Diego, CA, USA; Cat\# 302205; RRID AB_314235). Cell surface exhaustion markers were detected using PE-anti-human CD279 (PD1, Biolegend; Cat\# 621607; RRID AB_2832827), APC-anti-human CD366 (TIM3, Biolegend; Cat\# 345011; RRID AB_2561717) and Alexa Fluor ${ }^{\circledR}$ 647 anti-human CD233 (LAG3, Biolegend Cat\# 369303; RRID AB_2566479).

Intracellular staining was performed by fixation with $0.4 \%$ formaldehyde followed by permeabilization using PBS $+0.5 \%$ $\mathrm{BSA}+0.1 \%$ saponin. Cells were subsequently stained for $30 \mathrm{~min}$ on ice. CCR expression was detected using APC-conjugated antiDYKDDDDL (Biolegend, Cat\# 637307; RRID AB_2561496).

CD19-Fc binding studies were performed by addition of $0.5 \mu \mathrm{g} / \mathrm{mL}$ or $1.0 \mu \mathrm{g} / \mathrm{mL}$ CD19-Fc (contains human IgG1 Fc; Acro Biosystems, Newark, DE, USA; Cat\# CD9-H5251) to 0.5 x $10^{6}$ cells for 30 minutes on ice. Bound protein was detected with Alexa-Fluor ${ }^{\circledR} 647$ conjugated anti-human IgG (Jackson ImmunoResearch, Europe Ltd, Cat\# 109-605-006; RRID: AB_2337881). Since the scFv used in all CARs is of murine origin, this reagent does not bind directly to any CAR. To normalize binding to transduction efficiency (as determined by staining with anti-MYC antibody), the following formula was used.

$\%$ Normalized binding $=\%$ CD19-Fc binding to CAR T-cells $\%$ CD19-Fc binding to untransduced T-cells/\% transduced $\left(\mathrm{MYC}^{+}\right)$cells x 100 .

All gates were set using isotype control antibodies or fluorescence minus one controls. Where necessary, a viability stain (Zombie NIR ${ }^{\mathrm{TM}}$ Fixable Viability Kit, Biolegend; Cat\# 423105) was included and non-specific binding of the antibodies was limited by using an appropriate Fc blocking reagent prior to the staining steps.

All flow cytometry was performed using a FACSCalibur cytometer with CellQuest Pro software or BD LSRFortessa cytometer with BD FACSDiva software and data was analyzed using FlowJo, LLC.

\section{Enzyme-Linked Immunosorbent Assay}

Supernatants collected from co-culture of tumor cells with CAR Tcells were analyzed using a human interferon (IFN)- $\gamma$ (Cat\# 887316-76, RRID : AB_2575072) or human interleukin (IL)-2 (Cat\# 88-7025-76, RRID : AB_2574956) enzyme-linked immunosorbent assay (ELISA) as described by the manufacturers (ThermoFisher Scientific, Horsham, UK). In pooled re-stimulation assays, cytokine production was set to zero in each cycle after T-cell cultures failed.

\section{Cytotoxicity Assays}

Tumor cells were incubated with T-cells at specified effector to target (E:T) ratios. In the case of adherent targets, residual tumor cell viability was quantified using an MTT assay at the indicated time point. After removal of the supernatant and residual T-cells, MTT (Apollo Scientific; Cat\# BID2165) was added at $500 \mu \mathrm{g} / \mathrm{mL}$ in $\mathrm{D} 10$ medium for 40 minutes at $37^{\circ} \mathrm{C}$ and $5 \% \mathrm{CO}_{2}$. Formazan crystals were resuspended in DMSO and absorbance was measured at $560 \mathrm{~nm}$. Alternatively, tumor cell viability was monitored by luciferase assays. D-luciferin (R\&D Systems 
(Biotechne), Cat\# 122799) was added at $150 \mathrm{mg} / \mathrm{mL}$ immediately prior to luminescence reading. In each case, tumor cell viability was calculated using the following formula:

Absorbance or Luminescence of tumor cells cultured with Tcells/Absorbance or Luminescence of untreated monolayer alone x $100 \%$.

\section{Tumor Re-Stimulation Assays}

CD19-expressing LO68 target cells and CAR/pCAR T-cells were co-cultured in triplicates in a 24 -well plate at a 1:1 E:T ratio $(1 \mathrm{x}$ $10^{5}$ cells each) without further addition of cytokines. Supernatant was harvested after $24 \mathrm{~h}$ for cytokine analysis. At 72 hours tumor cell viability was assessed by MTT assay. If T-cells achieved more than $60 \%$ tumor cell destruction, they were restimulated on a fresh tumor monolayer. This process was repeated until T-cells failed to destroy $>60 \%$ of tumor cell monolayers.

\section{CAR Binding Studies - z-Movi}

CD19-engineered LO68 tumor cells were seeded in a $\mathrm{z}$-Movi microfluidic chip (Lumicks, Amsterdam, Netherlands) coated with poly-L-lysine and cultured for 16 hours. The next day, flow sorted CAR-T cells were serially flowed in the chips and incubated with the target cells for 5 minutes prior to initializing a 3-minute linear force ramp. During the force ramp, the $\mathrm{z}$-Movi device (Lumicks) captures a time series of images using a bright field microscope integrated into the platform. Detached cells were levitated towards the acoustic nodes, allowing the tracking of cells based on their XY positions. Changes in the Z-position results in a change in the diffraction pattern, which allows the distinction between cells adhered to the substrate and cells suspended to the acoustic nodes. This information is used to correlate cell detachment events with a specific rupture force. Cell detachment was acquired using z-Movi Tracking_v1.6.0 and post experiment image analysis was done using Cell Tracking offline analysis_v2.1. Data are presented as median acoustic force ( $\mathrm{rForce}$ ) which is the relative force required to elicit cell detachment and calibrated to $10 \mu \mathrm{M}$ polystyrene beads. Avidity score is calculated by the software as the ratio of the mean relative force ( $\mathrm{rForce}$ ) required to detach the CAR T-cells from an LO68-CD19 ${ }^{+}$tumor monolayer, when compared to untransduced controls. Thus, untransduced cells in each run have an avidity score of 1 .

\section{Evaluation of CAR T-Cell Number In Vivo}

After sacrifice, single cell suspensions were made of spleen by maceration through a cell strainer. Blood was collected by cardiac puncture from euthanised mice into Eppendorf tubes containing citrate-dextrose (Sigma) as anticoagulant. Red blood cells were lysed using 1X RBC Lysis Buffer (Biolegend) according to manufacturer's instructions. Cells were pelleted by centrifugation at $300 \mathrm{~g}$ for 5 minutes, resuspended in $1 \mathrm{~mL}$ and then stained with CountbrightTM beads (Cat\# 36950, Thermofisher), according to the manufacturers' instructions.

\section{In Vivo Xenograft Studies}

All in vivo experimentation adhered to U.K. Home Office guidelines, as specified in project licence number 70/7794 or P23115EBF and was approved by the King's College London animal welfare and ethical review body (AWERB). NOD SCID $\gamma_{c}$ null (NSG) mice were used for in vivo studies and were purchased from Charles River Laboratories (Harlow, UK). Mice were 6-10 weeks old when used for experiments. Similar numbers of male and female mice were used throughout. Nalm- 6 tumor cells were transduced with SFG ffLuc/RFP and were purified by flow sorting prior to engraftment in vivo by i.v. injection of $5 \times 10^{5}$ cells. Mice were allocated to experimental groups based on similar average tumor burden prior to treatment. Five days after Nalm-6 injection, CAR/pCAR Tcells were administered i.v. at a dose of $5 \times 10^{5}$ cells. To monitor tumor status, bioluminescence imaging (BLI) was performed using an IVIS Spectrum Imaging platform (PerkinElmer, Waltham, MA, USA) with Living Image software. Mice were injected i.p. with D-luciferin $[150 \mathrm{mg} / \mathrm{kg}$; R\&D Systems (Biotechne)] and imaged under isoflurane anesthesia after $20 \mathrm{~min}$. In all experiments, animals were inspected daily and weighed weekly.

\section{Statistical Analysis}

All data are derived from biological replicates involving independent donors unless otherwise indicated. For analysis of multiple groups, statistical analysis was performed using oneway or two-way ANOVA test (depending on the number of independent variables) followed by Tukey's multiple comparisons test. For non-parametrically distributed data, a Kruskal Wallis test was performed. Survival data were analyzed using a Log rank (Mantel-Cox) test. When only 2 groups were compared, a Student's t-test or Mann-Whitney test was performed, depending on normality of the data. Correlation testing was performed using a Spearman test. All statistical analyses were performed using GraphPad Prism version 9.1.

\section{RESULTS}

\section{Generation of a Panel of FMC63 Modified CARs With Specificity for CD19}

We focused on CARs that contain a CD28+CD3 $\zeta$ endodomain since CD19 expression is most commonly retained at the time of disease relapse, following infusion of these cells (16-18). To generate a panel of scFvs with varying ability to bind CD19, CDR3 of the $\mathrm{V}_{\mathrm{H}}$ domain within the CD19-specific FMC63 scFv was subjected to alanine scanning mutagenesis (Figure 1A). Chimeric antigen receptors were generated using an unmodified FMC63 scFv, dubbed F-2, in addition to these mutated scFvs, which were named according to mutation site within $V_{H}$ CDR3 (e.g. Y03, G01, G02, Y04 or Y05; Figures 1A, B) (23). Following retroviral transduction, cell surface expression of all CARs could be demonstrated in human T-cells by flow cytometry, taking advantage of an embedded MYC epitope tag within the CAR spacer domain. Despite similar cell surface expression levels (allowing for donor to donor variability; Figures 1C, D), the resulting panel of CARs presented a broad range of CD19-binding activity (Figure 1E). A representative example of this analysis is shown in Figure 1F. Cell surface CAR expression is specified in the upper section of each panel, as 

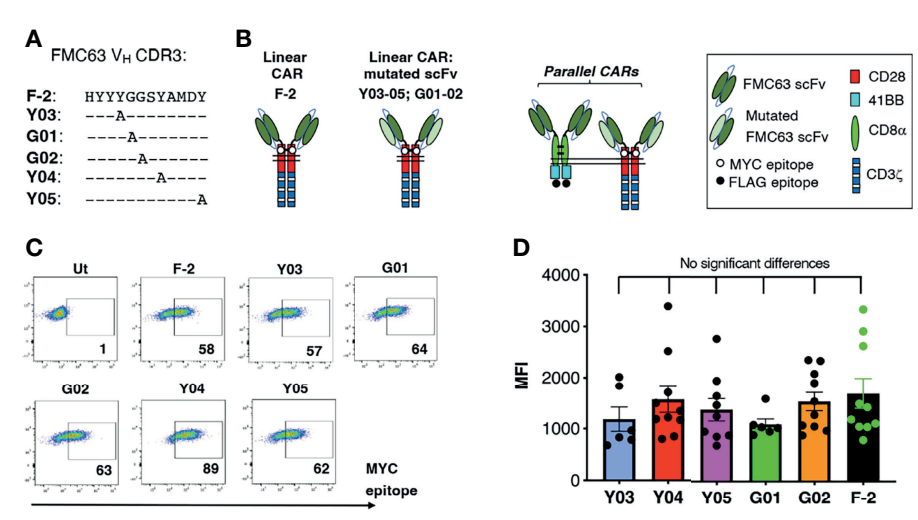

D
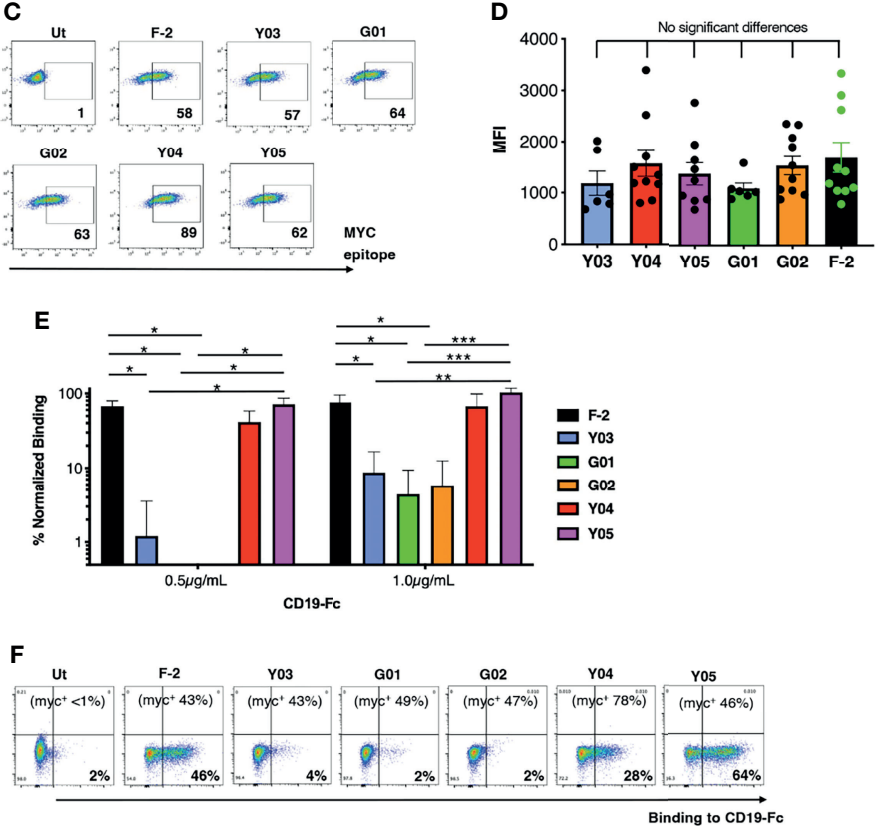

FIGURE 1 | Engineering of a panel of CD19-specific CARs. (A) The indicated mutations were introduced into the $V_{H}$ CDR3 region of an FMC63 scFv. (B) Unmodified and mutated FMC63 scFvs were used to target $2 \mathrm{G}(\mathrm{CD} 28+\mathrm{CD} 3 \zeta)$ CARs. The CAR containing the unmodified scFv was dubbed F-2 while CARs containing a modified scFv are named according to the amino acid within $V_{H} C D R 3$ that has been substituted with alanine. Structure of CD19 pCARs is also shown. Each consists of a CD28+CD3 $2 G$ CAR targeted by a mutated FMC63 scFv and co-expressed with a 4-1BB CCR in which an unmodified FMC63 scFv confers CD19 specificity. (C) Primary human T-cells were transduced with the indicated CD19-specific CARs and were analyzed by flow cytometry 5 days after gene transfer. Cells were incubated with 9 e10 antibody, which detects an embedded MYC epitope tag within the CAR, followed by goat anti-mouse secondary antibody. Data are representative of 7 independent replicate experiments. (D) Mean fluorescence intensity of CAR expression (mean $\pm \mathrm{SEM}, \mathrm{n}=6-10)$. Statistical analysis was performed using one-way ANOVA. (E) CAR T-cells were incubated with $\mathrm{CD} 19-\mathrm{Fc}$ protein $(0.5$ or $1.0 \mu \mathrm{g} / \mathrm{mL})$. Binding of Fc fusion protein was detected with Alexa-Fluor ${ }^{\circledR} 647 \mathrm{~V}_{\mathrm{H}}$ conjugated anti-human IgG. The indicated proportion of CARexpressing T-cells in each culture was determined by flow cytometry as described in $\mathrm{C}$ (mean +/- SEM, $n=3$ independent donor replicates). Following subtraction for nonspecific binding to untransduced cells, data were normalized for the \% CAR-expressing T-cells present. Statistical analysis was performed using one-way ANOVA. ${ }^{\star \star \star} p<$ $0.001 ;{ }^{* *} p<0.01 ;{ }^{\star} p<0.05$. One representative example of this analysis is also presented (F). Cell surface CAR expression was detected using anti-MYC antibody (percentage indicated in the top of each panel). Binding to a CD19-Fc fusion protein is shown for each CAR T-cell population.

detected using anti-MYC antibody. Panels show the binding of a CD19-IgG1 Fc fusion protein to each of these T-cell populations, which was detected using Alexa Fluor $647^{\circledR}$-conjugated antihuman IgG.

\section{Avidity Analysis of CD19-Specific CAR T-Cells}

We next ranked the avidity of these 2G CAR T-cells for CD19expressing tumor cells using z-Movi analysis (24). CAR T-cells were purified by flow sorting and then serially flowed on LO68$\mathrm{CD} 19^{+}$tumor cells (Figure S1) that had been pre-immobilized on microfluidic chips. Co-cultures were established for 5 minutes prior to the application of an acoustic force ramp to induce CAR T-cell detachment from the target cells. Figure 2A demonstrates the median percentage of $\mathrm{T}$-cells that remained bound to the $\mathrm{CD}_{1} 9^{+}$monolayer over the course of acoustic force ramp application. Curves were compiled from 11 separate runs using T-cells from 3 independent donors in which at least 1000 single cell observations were collected per run. The data demonstrate that these T-cells display a spectrum of avidities for CD19expressing target cells, with highly significant differences between all CARs tested. In Figure $\mathbf{2 B}$, an avidity score has been calculated as the ratio of the mean relative force (rForce) required to detach the CAR T-cells from an $\mathrm{LO} 68-\mathrm{CD} 19^{+}$ tumor monolayer, when compared to untransduced controls. Please note that only 3-4 technical replicates from 2 donors are included in this analysis, which may explain some differences relative avidity compared to data shown in Figure 2A (11 technical replicates from 3 donors). Figure $\mathbf{2 C}$ depicts a single representative run and illustrates the rForce required to detach individual CAR T-cells (each represented by a dot) during the application of the acoustic force ramp. The percentage of CAR 


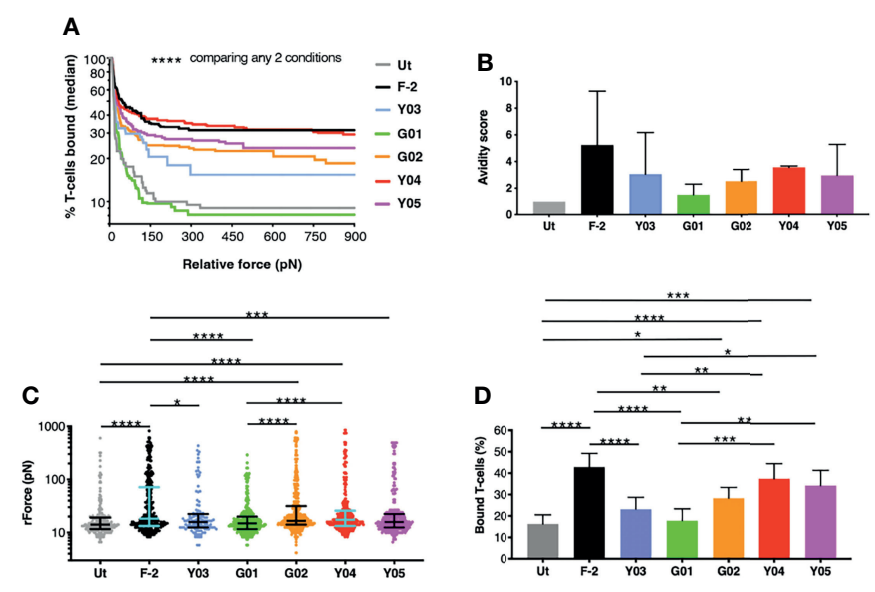

FIGURE 2 | z-Movi analysis of CAR T-cell avidity for CD19+ LO68 tumor cells. (A) T-cells were engineered to express the F-2 (CD28+CD3ל) CAR or 2G CAR derivates that contain a mutation in $V_{H}$ CDR3 region. After flow sorting to purity, CAR T-cells were incubated on CD19+ LO68 tumor cells within a z-Movi microfluidic chip. Increasing acoustic force was applied and the median percentage of bound T-cells was determined over time. Statistical analysis was performed using a Kruskal-Wallis test, comparing \% T-cells bound across the entire force ramp. (B) Avidity score represents the ratio of the mean relative force ( $r$ Force) per cell required to detach T-cells from the target CD19+ LO68 tumor monolayer, compared to untransduced (Ut.) T-cells (median + interquartile range, $n=3-4$ repeats from 2 independent donors). (C) Dot plot represents the rForce per cell required for detachment from the target cell monolayer. For clarity, a single representative run for one healthy donor is plotted in which each dot represents a single cell. Bars indicate median and interquartile range. Collectively, these dots generate the avidity curve shown in (A), meaning that all avidity curves are built with >1000 single cell observations per donor. Statistical analysis was performed using a Kruskal-Wallis test. (D) Bar plot depicting the percentage of T-cells bound to the target cell monolayer after applying a minimal rForce (210 pN) required to detach a median of $90 \%$ untransduced (Ut.) T-cells (mean \pm SEM of $n=11$ analyses incorporating 3 different healthy donors). Statistical analysis was performed using two-way ANOVA. ${ }^{* \star \star} p<$ $0.0001 ;{ }^{* \star} p<0.001 ;{ }^{* *} p<0.01 ;{ }^{*} p<0.05$.

T-cells that remain bound to the monolayer upon application of the minimal $\mathrm{rForce}$ required to dislodge a median of $90 \%$ untransduced cells is depicted in Figure 2D. Minimal rForce values for each CAR T-cell population correlated with \% normalized binding of these cells to CD19-Fc (Pearson $r=$ $0.85(p=0.015)$ and $r=0.83(p=0.021)$ at $0.5 \mu \mathrm{g} / \mathrm{mL}$ and $1.0 \mu \mathrm{g} / \mathrm{mL}$ respectively). Collectively, these data show that Y03 and G01-expressing CAR T-cells exhibited markedly reduced target cell avidity compared to F-2-expressing cells, close to (Y03) or comparable (G01) to that of untransduced T-cells. By contrast, the avidity of Y04-expressing CAR T-cells was similar to F-2 cells, while intermediate avidity was seen with Y05 and G02 CAR T-cells.

\section{Functional Comparison of CD19-Specific CAR T-Cells}

Next, we compared the in vitro anti-tumor activity of this panel of CD19-specific CARs. All five scFv modified CARs mediated the cytotoxic destruction of the malignant B-cell lines, Nalm-6 (Figures 3A, B) and Raji (Figures 3C, D). Expression of CD19 on these tumor cells in addition to CD19-engineered LO68 cells is shown in Figure S2. This was accompanied by production of IFN- $\gamma$ (Nalm-6, Figure 3E; Raji, Figure 3F) and IL-2 (Nalm-6, Figure 3G; Raji, Figure 3H). Impaired cytotoxicity against Raji cells and reduced cytokine production was noted for T-cells that expressed the G01 CAR, in keeping with its very weak CD19 binding activity and poor avidity for LO68-CD19 tumor cells. Activated T-cells that expressed the Y03 CAR also produced lower levels of IFN- $\gamma$, in accordance with the reduced ability of this CAR to bind CD19 and lowered avidity of these cells for LO68-CD19 cells. However, the G02 CAR demonstrated a trend towards enhanced cytokine release when compared to the parental F-2 CAR, despite low binding of CD19-Fc. Notably, G02 CAR T-cells retained an intermediate avidity for CD19expressing LO68 tumor cells, a property that was shared with Y05 CAR T-cells.

\section{Comparison of In Vitro Function of CD19-Specific CARs and Parallel CARs}

We have recently demonstrated superior anti-tumor function of pCAR T-cells, in which a CD28-containing $2 \mathrm{G}$ CAR is coexpressed with a 4-1BB-containing CCR (10). To test the applicability of the pCAR platform to targeting of CD19, we co-expressed each of the mutant scFv-based 2G CARs described above with a 4-1BB CCR that contains an unmodified FMC63 $\mathrm{scFv}$. We selected this arrangement since we have found that excessive affinity (e.g. low picomolar $\mathrm{k}_{\mathrm{d}}$ ) compromises CAR, but not CCR function, in the context of a PCAR (unpublished data). The FMC63 scFv has high affinity for CD19 whereas all mutated $\mathrm{scFv}$ derivatives mediate similar or lower avidity for CD19expressing target cells. Parallel CARs are named as $p C A R-X / Y$ where $\mathrm{X}$ and $\mathrm{Y}$ respectively are abbreviations for the targeting moiety used in the CAR (i.e. mutated FMC63 scFv such as G02) and CCR (i.e. unmodified FMC63 scFv, abbreviated as F) (Figure 1C). Cell surface 1:1 co-expression of CAR and CCR components was demonstrated by flow cytometry (Figure 4A).

Next, we compared the anti-tumor activity of these CAR and pCAR T-cells in vitro. In cytotoxicity assays performed at a range 


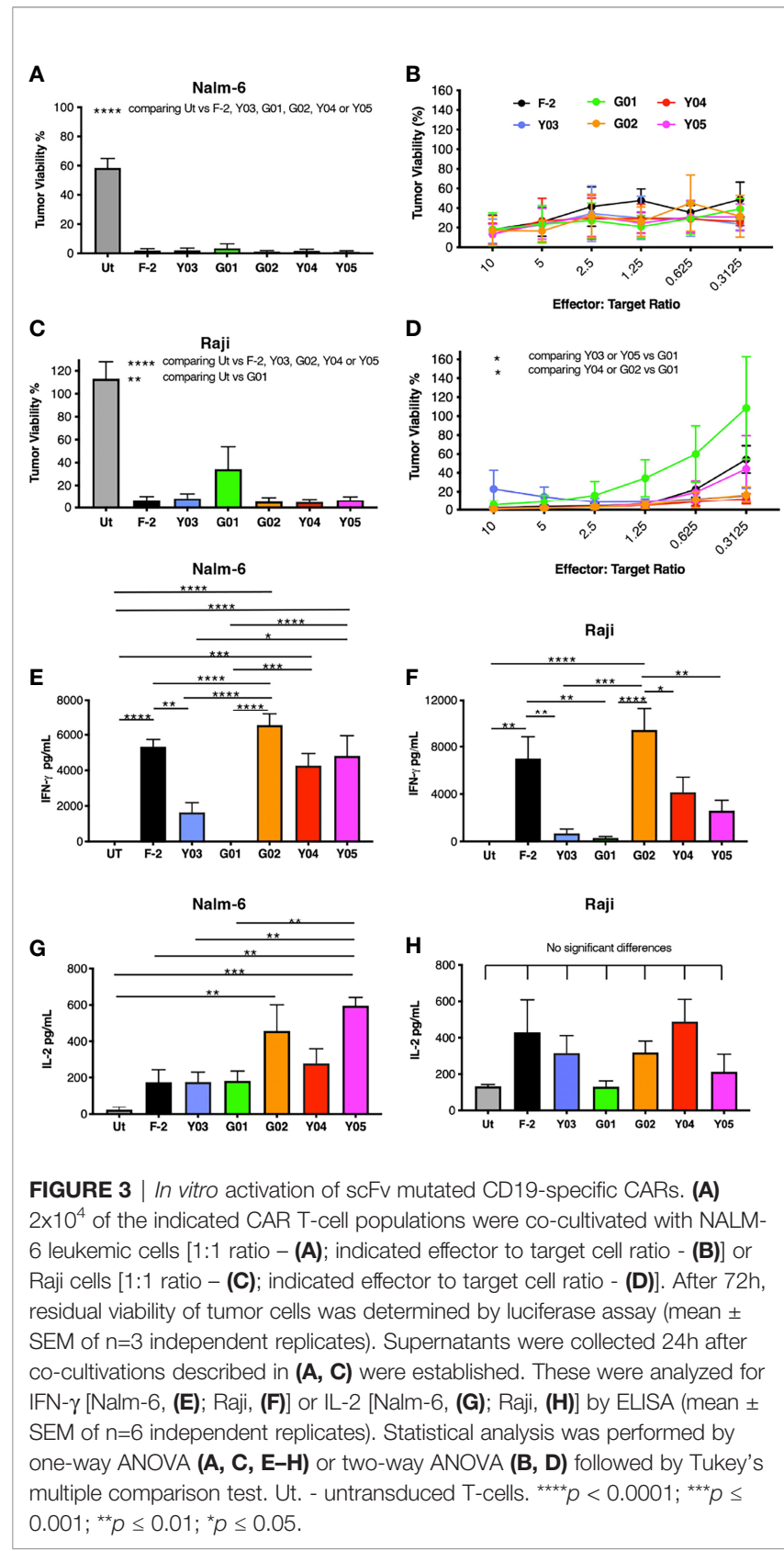

of low effector to target ratios, the G02 CAR demonstrated significantly enhanced killing activity against Nalm-6 leukemic cells compared to the parental F-2 CAR (Figure 4B). Cytolytic activity of all pCAR T-cell populations was similar or superior compared to the corresponding mutant scFv-based $2 \mathrm{G} \mathrm{CAR}$, or the F-2 CAR (Figure 4B). We have previously shown that a docking effect of the CCR can potentiate cytolytic activity of some, but not all pCARs, when compared to the parental CAR (10). Analysis of immune synapse formation by these T-cell populations could help to uncover mechanisms that may explain this variable response of pCAR T-cells. Parallel CAR T-cells strongly outperformed those that expressed the corresponding mutant scFv-based CAR or F-2 in tumor re-stimulation assays, maintaining cytolytic activity over a significantly greater number of stimulation cycles in every case (Figure 5A). Strikingly, pCAR T-cells produced significantly more IL-2 over early tumor restimulation cycles, unlike CAR T-cells in which specificity was conferred by the same mutated scFv or F-2 (Figure 5B). A trend towards more sustained IFN- $\gamma$ production upon iterative tumor re-stimulation was also observed for pCAR T-cells, compared to $\mathrm{T}$-cells that expressed the corresponding CAR or F-2 (Figure 5C).

Given these findings, the best performing CARs and pCARs (Y04, Y05, G02 and derived pCARs) were advanced for further study, making comparison with the F-2 2G CAR and untransduced T-cells. Upon iterative tumor cell re-stimulation in the absence of exogenous cytokine support, all three pCARs mediated significantly greater T-cell expansion when compared to CAR alone or F-2 (Figure 6A). Analysis of exhaustion marker expression demonstrated that PD1 was highly upregulated when either CAR or pCAR T-cells were stimulated on LO68-CD19 tumor monolayers (Figure 6B). However, PD1 levels after one stimulation cycle were significantly lower on pCAR T-cells compared to the matched CAR and this also remained the case for $p C A R-G 02 / F$ pCAR T-cells after the third stimulation cycle (Figure 6B). While no differences between CAR and matched

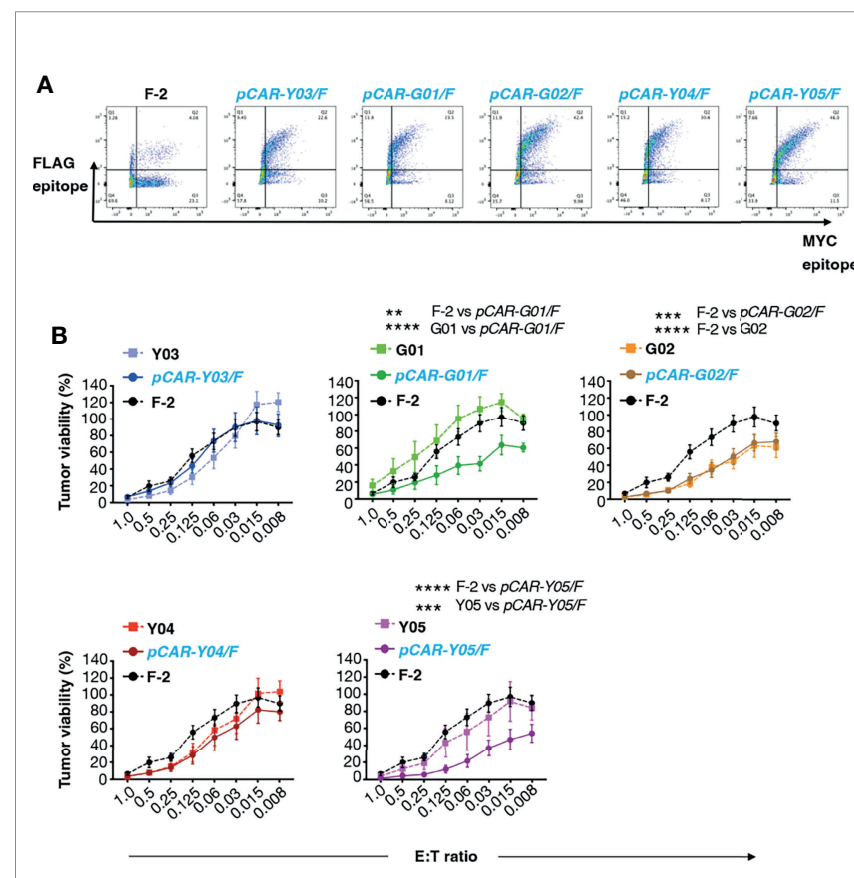

FIGURE 4 | Evaluation of cytolytic activity of pCARs targeted against CD19. (A) Human T-cells were engineered by retroviral transduction to express the indicated pCARs, or F-2 CAR as control. Permeabilized T-cells were incubated with antibodies directed against embedded MYC (CAR) and FLAG (CCR) epitope tags and then analyzed by flow cytometry. Data are representative of three independent replicate experiments. (B) CAR or pCAR T-cells were co-cultivated with ffLuc/RFP ${ }^{+}$Nalm-6 leukemia cells at the indicated $\mathrm{E}: T$ ratio. Target viability was quantified after $72 \mathrm{~h}$ ( $m$ mean \pm SEM, $n=7$ replicates from 5 donors). Statistical analysis was performed using two-way ANOVA. ${ }^{* \star} p<0.01 ;{ }^{* \star *} p<0.001 ;{ }^{* \star *} p<0.0001$. 

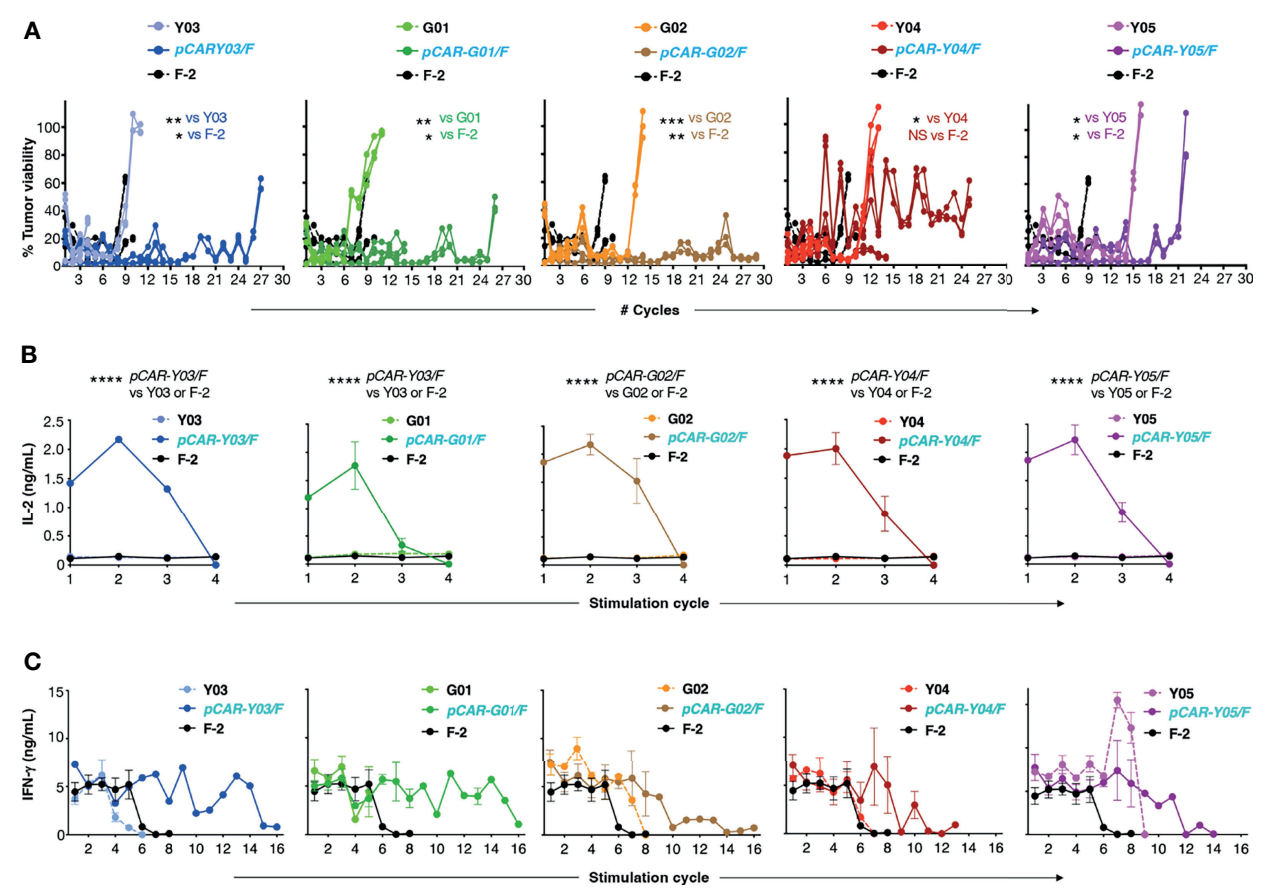

FIGURE 5 | Re-stimulation of CD19-targeted CAR and pCAR T-cells. (A) T-cells were engineered to express the indicated CARs or pCARs. $1 \times 10^{5}$ of the indicated transduced CAR or pCAR T-cells were co-cultivated in triplicate in a $1 \mathrm{~mL}$ volume with an equal number of LO68-CD19+ tumor cells. After $72 \mathrm{~h}$, T-cells were transferred to a fresh monolayer of CD19+ LO68 cells and viability of the original tumor monolayer was determined by MTT assay. Plots indicate tumor cell viability at each stimulation cycle. Cultures were terminated when tumor cell killing was $<60 \%$ or if T-cells could not be recovered. Statistical analysis was performed using an unpaired Student's $t$ test comparing the number of stimulation cycles achieved by each pCAR compared to the indicated CAR. Supernatant was collected 24h after the initiation of each stimulation cycle and was analyzed by ELISA for IL-2 [mean +/- SEM, n=2; (B)] or IFN- $\gamma$ [mean +/- SEM, $n=6$; (C)] (mean +/- SEM of duplicate independent cultures). Statistical analysis was performed using two-way ANOVA. ${ }^{* * *} p=<0.0001 ;{ }^{* \star *} p=\leq 0.001 ;{ }^{* *} p=\leq 0.01 ;{ }^{*} p<0.05$.

pCAR T-cells were noted for either LAG3 or TIM3 expression (Figure 6B), repeatedly stimulated $p C A R-Y 05 / F$ and $p C A R-G 02 /$ $F$ T-cell cultures demonstrated a trend towards reduced numbers of cells that co-expressed all three exhaustion markers (triple pos.; Figure 6C).

\section{In Vivo Comparison of CD19-Specific CAR and pCAR T-Cells}

In vivo anti-tumor activity of the advanced panel of CAR and pCAR T-cells was compared with the commonly used CD $19^{+}$ Nalm-6 xenograft model of B-cell acute lymphoblastic leukemia (B-ALL), using BLI to monitor disease status. The experimental scheme used in the treatment of mice with an established disease burden is indicated in Figure 7A. Pooled results of two independent experiments are shown. CAR and pCAR T-cell transduction efficiency was determined 24 hours prior to i.v. infusion by flow cytometry and the transduction efficiency was normalized across groups by the addition of untransduced Tcells. Following CAR/pCAR T-cell treatment, all animals were monitored by BLI weekly. At the modest CAR/pCAR T-cell dose employed ( $5 \times 10^{5}$ cells), F-2 CAR T-cells caused a delay in leukemic progression compared to PBS, while the two intermediate avidity 2G CAR derivatives (G02 and Y05) achieved a further significant improvement in disease control
(Figure 7B). Importantly, all pCARs achieved significantly enhanced disease control (Figure 7B) and extended survival (Figure 7C) when compared to F-2 or their CAR of origin. Survival was greatest when pCARs contained an intermediate avidity CAR (e.g. G02 or Y05), rather than a high avidity CAR (e.g. Y04), reaching significance in the comparison between $p C A R-G 02 / F$ and $p C A R-Y 04 / F$. Clinical evidence of CAR Tcell-induced toxicity was not apparent in any treatment group, nor was non-tumor-related weight loss observed following treatment (Figure S3). It should be noted however that cytokine release syndrome cannot be accurately modeled in NSG mice owing to the lack of fully functional macrophages in these animals (25). Parallel CAR T-cells demonstrated enhanced in vivo persistence compared to control F-2 $2 \mathrm{G}$ CAR T-cells (Figure S4). Together, these data demonstrate that the pCAR platform enables the delivery of superior anti-tumor activity using $\mathrm{scFv}$ targeting moieties and across a broad range of relative CAR/CCR binding strengths.

\section{DISCUSSION}

While the clinical success of CD19-specific CAR T-cells provides tremendous encouragement, there remains a need to further 
Y
Y04 vs $P C A R-Y 04 / F(*)$
F-2 $P C A R-Y 04 F(*)$

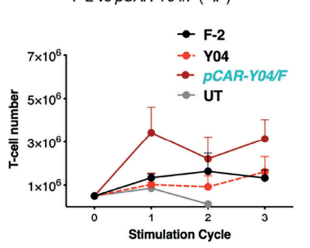

B
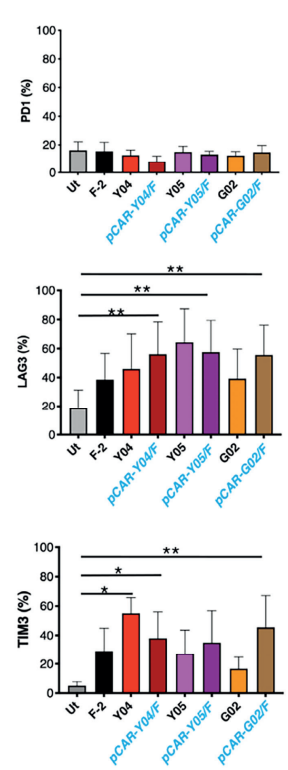

C

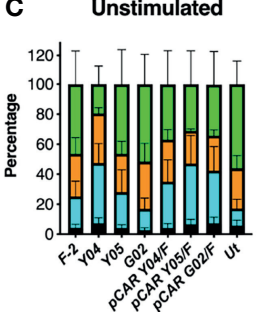

comparing Y05 vs $P C A R-Y 05 / F(*)$
comparing F-2 vs $P C A R-Y 05 / F(* *)$

comparing G02 vs $P C A R-G 02 / F(*)$ comparing F-2 vs $P C A R-G O 2 F(* * *)$
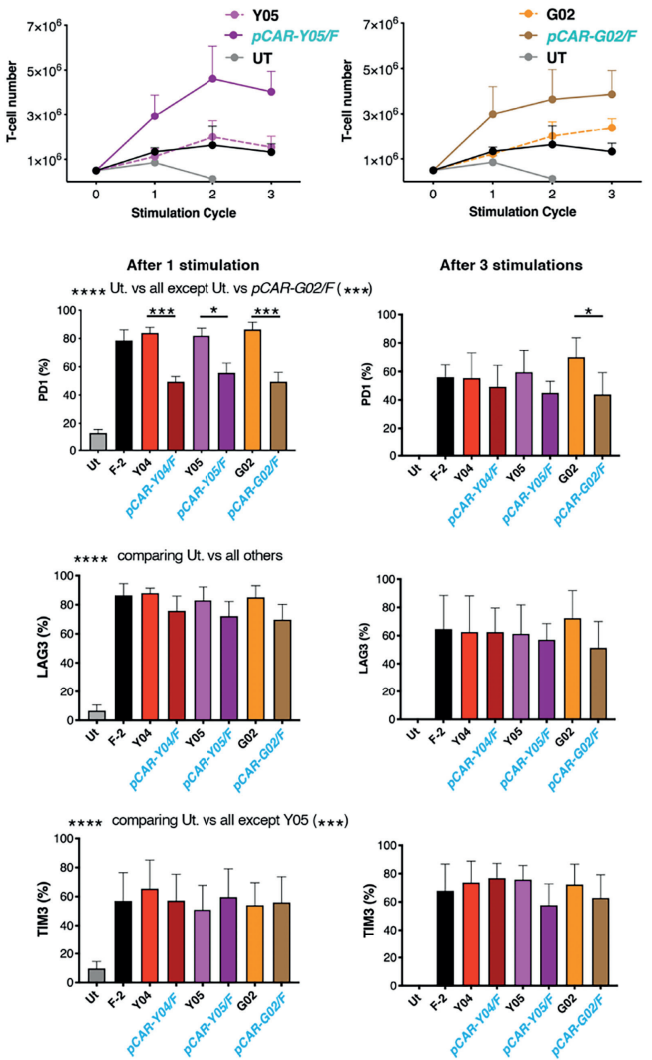

1 Stimulation Cycle

3 Stimulation Cycles

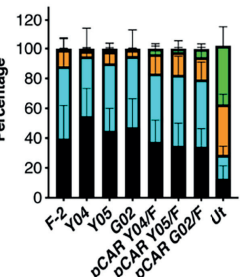

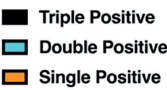

$\square$ Negative

FIGURE 6 | Expansion of tumor re-stimulated pCAR T-cells is accompanied by reduced exhaustion marker expression. (A) T-cells were engineered to express the indicated CARs or pCARs. $5 \times 10^{5}$ of the indicated transduced CAR or pCAR T-cells were co-cultivated with $2.5 \times 10^{5}$ LO68-CD19 tumor cells. After $72 \mathrm{~h}$, T-cells were harvested, counted and transferred to a new tumor monolayer. Data show T-cell number at the time of each stimulation cycle (mean \pm SEM, $\mathrm{n}=3-4)$. Statistical analysis was performed using an unpaired Student $t$-test, comparing cell number between the indicated groups over stimulation cycles 1 -3. ${ }^{\star} p<0.05$; ${ }^{\star \star} p<0.01$. (B) T-cells described in A that were unstimulated or 72 hours after the indicated number of stimulation (stim.) cycles were analyzed by flow cytometry for expression of PD1, LAG3 and TIM3. Statistical analysis was performed using two-way ANOVA with Tukey's multiple comparisons. ${ }^{*} p<0.05 ;{ }^{* \star} p<0.01 ;{ }^{* \star \star} p<0.001 ;{ }^{* \star \star \star} p<$ 0.0001. (C) Bar graph of exhaustion marker data shown in (B) Segments indicate cells that were negative or were triple, double or single positive for the exhaustion markers PD1, LAG3 and/or TIM3 (mean \pm SEM).

improve remission rates and durability of disease control. We hypothesized that this may be achieved using pCAR T-cells that harness dual co-stimulation by CD28 and 4-1BB. Parallel CARs consist of the stoichiometric co-expression of a $\mathrm{CD} 28+\mathrm{CD} 3 \zeta 2 \mathrm{G}$ CAR with a $4-1 \mathrm{BB}$ containing CCR. Using a range of tumor models, we have previously demonstrated that pCAR T-cells outperform linear CARs that contain one or two co-stimulatory domains or alternative configurations that place one co-stimulatory domain away from the plasma membrane (10). Parallel CAR T-cells also undergo reduced apoptosis upon iterative tumor re-stimulation (10). Broadly similar results are obtained if co-stimulatory modules are switched between CAR and CCR (data not shown). In keeping with these advantageous properties of pCAR T-cells, administration of an anti-4-1BB antibody boosts anti-tumor activity of CD28-containing 2G CAR T-cells (26). Moreover, provision of either CD28 or $4-1 \mathrm{BB}$ 


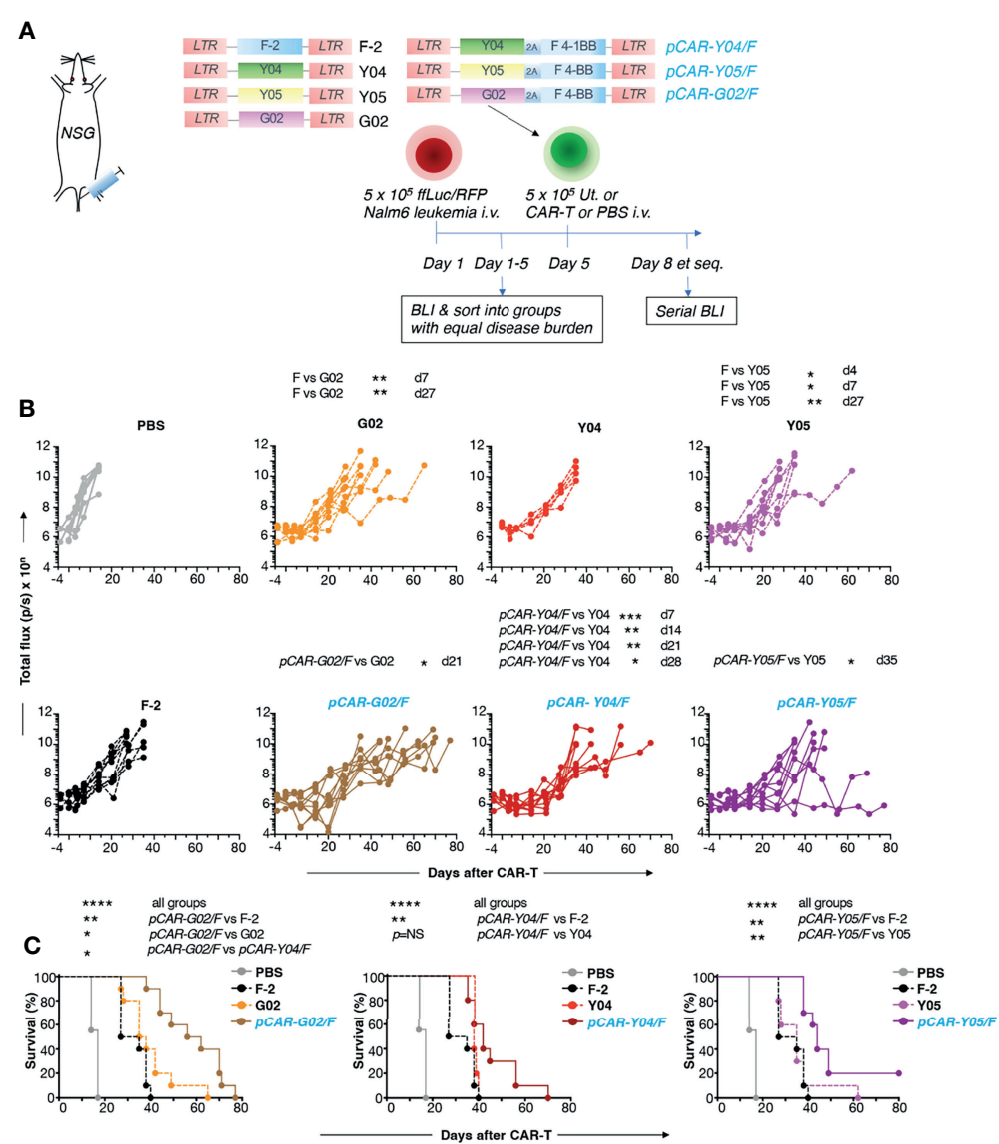

FIGURE 7 | In vivo evaluation of pCARs targeted against CD19. (A) NSG mice ( $\mathrm{n}=5-10$ per group) were inoculated i.v. with $5 \times 10^{5}$ ffLuc/RFP ${ }^{+}$Nalm-6 cells. On day 5 , mice with established leukemia were treated i.v. with $5 \times 10^{5}$ CAR or pCAR T-cells or PBS. (B) Tumor burden in individual mice following treatment was monitored by BLI. Data are pooled from 2 donors. Statistical analysis was performed using an unpaired Student $t$ test comparing the indicated CARs/pCARs. (C) Survival curves of mice. Statistical analysis was performed using a Log-rank (Mantel-Cox) test, comparing the indicated groups. ${ }^{\star} p<0.05 ;{ }^{\star \star} p<0.01 ;{ }^{* \star *} p<0.001 ;{ }^{* \star *} p<0.0001$.

co-stimulation to an activated T-cell in cis is markedly more potent than when either is provided in trans (27).

In this study, we have evaluated the suitability of the pCAR platform for the treatment of CD19-expressing malignancy with a view to identifying an optimal candidate for clinical advancement. The FMC63 scFv is most commonly used in CD19 CARs and has low nanomolar affinity for this antigen $\left(\mathrm{K}_{\mathrm{a}} 2.3 \times 10^{-9}\right)(23)$. Some prior studies have indicated that high affinity CARs retain satisfactory anti-tumor activity, whereas lower affinity derivatives have superior capacity to discriminate between tumor cells that express high levels of target antigen and normal cells in which target antigen is found at lower levels (2830). However, studies with TCRs have indicated that that there is an affinity ceiling above which increased binding strength adversely affects T-cell response (31-33). This phenomenon has also been reported for some high affinity CARs which retain and sequester target ligand efficiently, potentially preventing serial killing of tumor cells (34). Clinical evidence in support of this concept arises from a recent trial involving
B-ALL. Patients were treated with CAR T-cells that had $>40$-fold reduction in affinity for CD19 compared to FMC63 CARs. The lower affinity CAR demonstrated superior performance in preclinical testing and maintained excellent efficacy, but without any severe toxicity when evaluated in man (35). For this reason, we undertook mutagenesis of the FMC63 scFv in order to generate a panel of derivates that encompassed a spectrum of CD19 binding strengths.

Commonly, CAR affinity is inferred from biophysical studies performed using a soluble form of the targeting moiety, such as an scFv-Fc fusion (35). However, this is a time-consuming undertaking which requires expression and purification of the panel of scFvs under study prior to undertaking binding studies, for example using surface plasmon resonance biosensors such as the BIAcore protein interaction platform. While this provides useful information regarding the $\mathrm{scFv}$ itself, this analysis does not consider the geography of chimeric antigen receptor or target antigen expression within the cell membrane, nor the influence of secondary binding interactions mediated by other 
pro-adhesive molecules. Accordingly, some studies of scFv affinity have proven poorly predictive of CAR function (36). To measure the global strength of interaction between our panel of CD19-specific CAR T-cells and a target cell that expresses this antigen, we undertook z-Movi avidity testing. These CAR T-cells demonstrated a spectrum of avidities with strongest interaction mediated by the original F-2 CAR and the Y04 mutant. The G01 and Y03 mutants had low avidity in agreement with CD19binding studies. Intermediate avidity was observed with the Y05 and G02 mutants, despite the greater ability of the former to bind CD19-Fc. Notably, both of these CAR T-cells demonstrated significantly greater in vivo anti-tumor activity in the Nalm-6 leukemic xenograft model, when compared to the parental F-2 CAR. Conceptually, CAR T-cells with intermediate avidity may have a superior ability to dock transiently on target cells, enabling serial tumor cell killing (37) and a reduction in deleterious effects of over-activation, such as exhaustion and activation-induced cell death (38). Despite its poor ability to bind CD19, the superior target cell avidity and anti-tumor activity of the G02 CAR suggests that it can recruit additional adhesive mechanisms to achieve productive target cell engagement, perhaps via enhanced immune synapse formation.

We proceeded to generate $\mathrm{PCAR}$ derivatives of these CARs by co-expression of a CCR in which an unmodified FMC63 scFv was coupled via a $\operatorname{CD} 8 \alpha$ spacer and transmembrane domain to a 4$1 \mathrm{BB}$ endodomain. As observed with previously described pCARs (10), all CD19-specific pCAR T-cells achieved markedly enhanced tumor re-stimulation activity, maintained capacity to produce IL-2 over repeated stimulation and reduced expression of exhaustion markers, most notably PD1. Enhanced cytokine production by pCAR compared to second-generation CAR T-cells is consistent with the effects of dual co-stimulation via CD28 and 4-1BB, as described previously (39). Conversion to a pCAR system also enhanced the cytolytic activity of G01 CAR T-cells, despite poor CD19 binding and low avidity of these T-cells for CD19expressing targets. This suggests that the CCR may contribute to target cell docking under some circumstances, as described for other pCARs (10). In keeping with this, recently published data indicate that co-expression of a CCR together with a CAR leads to increased functional avidity and enhanced sensitivity to detect tumor cells that express low levels of CAR target antigen (40). When administered at a low dose to mice with an established Nalm-6 leukemic burden, all pCAR T-cells significantly outperformed their CAR counterparts, or the parental F-2 CAR, in which an unmodified FMC63 scFv conferred CD19 specificity. Accordingly, disease progression was delayed and survival enhanced, without significant toxicity. In particular, the $p C A R$ $G 02 / F$ pCAR achieved the greatest survival advantage, suggesting that intermediate CAR avidity may also be a favorable attribute of pCAR T-cells, at least under conditions of high CD19 expression. To further characterize this platform, additional studies that compare anti-tumor activity of all configurations of high and/or low avidity targeting moieties in the CAR and CCR would be useful. Additional testing of T-cells from a larger donor panel, including patients with B-cell malignancy, and testing on target cells with different antigen densities would provide further useful confirmatory information. Taken together, these data support the clinical evaluation of CD19 pCAR immunotherapy in patients with relapsed refractory B-cell malignancy.

\section{DATA AVAILABILITY STATEMENT}

Publicly available datasets were analyzed in this study. This data can be found here: https://www.ncbi.nlm.nih.gov/ nuccore/, HM852952.1.

\section{ETHICS STATEMENT}

The studies involving human participants were reviewed and approved by London Bridge Research Ethics Committee and Research Ethics Committee 3, West of Scotland. The patients/ participants provided their written informed consent to participate in this study. The animal study was reviewed and approved by King's College London Animal Welfare and Ethical Review Body.

\section{AUTHOR CONTRIBUTIONS}

Conceptualization, JM. Experimental work, LH, KD, DL-Y, AA, and DD. Methodology and data analysis, all authors. Project Oversight AC, RD, and JM. Writing JM. Review and editing, all authors. All authors contributed to the article and approved the submitted version.

\section{FUNDING}

This work was supported by Leucid Bio, the British Lung Foundation (MESOUK18-2 INT 1), the Medical Research Council (MR/R001936/1), the Experimental Cancer Medicine Centre at King's College London, the King's Health Partners/ King's College London Cancer Research UK Cancer Centre and by the National Institute for Health Research (NIHR) Biomedical Research Centre based at Guy's and St Thomas' NHS Foundation Trust and King's College London (IS-BRC-1215-20006).

\section{ACKNOWLEDGMENTS}

We thank A Adami and Dr A Schurich for critical review of the manuscript and scientific colleagues for provision of materials.

\section{SUPPLEMENTARY MATERIAL}

The Supplementary Material for this article can be found online at: https://www.frontiersin.org/articles/10.3389/fimmu.2022. 836549/full\#supplementary-material 


\section{REFERENCES}

1. Yu JX, Upadhaya S, Tatake R, Barkalow F, Hubbard-Lucey VM. Cancer Cell Therapies: The Clinical Trial Landscape. Nat Rev Drug Discov (2020) 19 (9):583-4. doi: 10.1038/d41573-020-00099-9

2. Finney HM, Lawson AD, Bebbington CR, Weir AN. Chimeric Receptors Providing Both Primary and Costimulatory Signaling in T Cells From a Single Gene Product. J Immunol (1998) 161(6):2791-7.

3. Finney HM, Akbar AN, Lawson AD. Activation of Resting Human Primary T Cells With Chimeric Receptors: Costimulation From CD28, Inducible Costimulator, CD134, and CD137 in Series With Signals From the TCR Zeta Chain. J Immunol (2004) 172(1):104-13. doi: 10.4049/jimmunol. 172.1.104

4. Maher J, Brentjens RJ, Gunset G, Riviere I, Sadelain M. Human TLymphocyte Cytotoxicity and Proliferation Directed by a Single Chimeric TCRzeta /CD28 Receptor. Nat Biotechnol (2002) 20(1):70-5. doi: 10.1038/ nbt0102-70

5. Imai C, Mihara K, Andreansky M, Nicholson IC, Pui CH, Geiger TL, et al. Chimeric Receptors With 4-1BB Signaling Capacity Provoke Potent Cytotoxicity Against Acute Lymphoblastic Leukemia. Leukemia (2004) 18 (4):676-84. doi: 10.1038/sj.leu.2403302

6. Melero I, Bach N, Hellstrom KE, Aruffo A, Mittler RS, Chen L. Amplification of Tumor Immunity by Gene Transfer of the Co-Stimulatory 4-1BB Ligand: Synergy With the CD28 Co-Stimulatory Pathway. Eur J Immunol (1998) 28 (3):1116-21. doi: 10.1002/(SICI)1521-4141(199803)28:03<1116::AIDIMMU1116>3.0.CO;2-A

7. Wen T, Bukczynski J, Watts TH. 4-1BB Ligand-Mediated Costimulation of Human T Cells Induces CD4 and CD8 T Cell Expansion, Cytokine Production, and the Development of Cytolytic Effector Function. J Immunol (2002) 168(10):4897-906. doi: 10.4049/jimmunol.168.10.4897

8. Maus MV, Thomas AK, Leonard DG, Allman D, Addya K, Schlienger K, et al. Ex Vivo Expansion of Polyclonal and Antigen-Specific Cytotoxic $\mathrm{T}$ Lymphocytes by Artificial APCs Expressing Ligands for the T-Cell Receptor, CD28 and 4-1BB. Nat Biotechnol (2002) 20(2):143-8. doi: 10.1038/nbt0202-143

9. Salter AI, Ivey RG, Kennedy JJ, Voillet V, Rajan A, Alderman EJ, et al. Phosphoproteomic Analysis of Chimeric Antigen Receptor Signaling Reveals Kinetic and Quantitative Differences That Affect Cell Function. Sci Signal (2018) 11(544):eaat6753. doi: 10.1126/scisignal.aat6753

10. Muliaditan T, Halim L, Whilding LM, Draper B, Achkova DY, Kausar F, et al. Synergistic T-Cell Signaling by 41BB and CD28 Is Optimally Achieved by Membrane Proximal Positioning Within Parallel Chimeric Antigen Receptors. Cell Rep Med (2021) 2:100457. doi: 10.1016/j.xcrm.2021.100457

11. Pule MA, Straathof KC, Dotti G, Heslop HE, Rooney CM, Brenner MK. A Chimeric T Cell Antigen Receptor That Augments Cytokine Release and Supports Clonal Expansion of Primary Human T Cells. Mol Ther (2005) 12 (5):933-41. doi: 10.1016/j.ymthe.2005.04.016

12. Kloss CC, Condomines M, Cartellieri M, Bachmann M, Sadelain M. Combinatorial Antigen Recognition With Balanced Signaling Promotes Selective Tumor Eradication by Engineered T Cells. Nat Biotechnol (2013) 31(1):71-5. doi: 10.1038/nbt.2459

13. Adami A, Maher J. An Overview of CAR T-Cell Clinical Trial Activity to 2021. Immunother Adv (2021) 1(1):Itab004. doi: 10.1093/immadv/ltab004

14. Cappell KM, Kochenderfer JN. A Comparison of Chimeric Antigen Receptors Containing CD28 Versus 4-1BB Costimulatory Domains. Nat Rev Clin Oncol (2021) 18:715-27. doi: 10.1038/s41571-021-00530-z

15. Spiegel JY, Patel S, Muffly L, Hossain NM, Oak J, Baird JH, et al. CAR T Cells With Dual Targeting of CD19 and CD22 in Adult Patients With Recurrent or Refractory B Cell Malignancies: A Phase 1 Trial. Nat Med (2021) 27(8):141931. doi: 10.1038/s41591-021-01436-0

16. Xu X, Sun Q, Liang X, Chen Z, Zhang X, Zhou X, et al. Mechanisms of Relapse After CD19 CAR T-Cell Therapy for Acute Lymphoblastic Leukemia and Its Prevention and Treatment Strategies. Front Immunol (2019) 10:2664. doi: 10.3389/fimmu.2019.02664

17. Plaks V, Rossi JM, Chou J, Wang L, Poddar S, Han G, et al. CD19 Target Evasion as a Mechanism of Relapse in Large B-Cell Lymphoma Treated With Axicabtagene Ciloleucel. Blood (2021) 138:1081-5. doi: 10.1182/ blood.2021010930
18. Martino M, Alati C, Canale FA, Musuraca G, Martinelli G, Cerchione C. A Review of Clinical Outcomes of CAR T-Cell Therapies for B-Acute Lymphoblastic Leukemia. Int J Mol Sci (2021) 22(4):2150. doi: 10.3390/ ijms 22042150

19. Kochenderfer JN, Feldman SA, Zhao Y, Xu H, Black MA, Morgan RA, et al. Construction and Preclinical Evaluation of an Anti-CD19 Chimeric Antigen Receptor. J Immunother (2009) 32(7):689-702. doi: 10.1097/CJI.0b013e3181ac6138

20. Whilding LM, Parente-Pereira AC, Zabinski T, Davies DM, Petrovic RMG, Kao YV, et al. Targeting of Aberrant Alphavbeta6 Integrin Expression in Solid Tumors Using Chimeric Antigen Receptor-Engineered T Cells. Mol Ther (2017) 25(1):259-73. doi: 10.1016/j.ymthe.2016.10.012

21. Emami-Shahri N, Foster J, Kashani R, Gazinska P, Cook C, Sosabowski J, et al. Clinically Compliant Spatial and Temporal Imaging of Chimeric Antigen Receptor T-Cells. Nat Commun (2018) 9(1):1081. doi: 10.1038/s41467-01803524-1

22. Whilding LM, Halim L, Draper B, Parente-Pereira AC, Zabinski T, Davies DM, et al. CAR T-Cells Targeting the Integrin Alphavbeta6 and CoExpressing the Chemokine Receptor CXCR2 Demonstrate Enhanced Homing and Efficacy Against Several Solid Malignancies. Cancers (Basel) (2019) 11(5):.674 doi: 10.3390/cancers11050674

23. Nicholson IC, Lenton KA, Little DJ, Decorso T, Lee FT, Scott AM, et al. Construction and Characterisation of a Functional CD19 Specific Single Chain Fv Fragment for Immunotherapy of B Lineage Leukaemia and Lymphoma. Mol Immunol (1997) 34(16-17):1157-65. doi: 10.1016/s01615890(97)00144-2

24. de Larrea CF, Staehr M, Lopez AV, Ng KY, Chen Y, Godfrey WD, et al. Defining an Optimal Dual-Targeted CAR T-Cell Therapy Approach Simultaneously Targeting BCMA and GPRC5D to Prevent BCMA EscapeDriven Relapse in Multiple Myeloma. Blood Cancer Discov (2020) 1(2):14654. doi: 10.1158/2643-3230.bcd-20-0020

25. van der Stegen SJ, Davies DM, Wilkie S, Foster J, Sosabowski JK, Burnet J, et al. Preclinical In Vivo Modeling of Cytokine Release Syndrome Induced by ErbB-Retargeted Human T Cells: Identifying a Window of Therapeutic Opportunity? J Immunol (2013) 191(9):4589-98. doi: 10.4049/jimmunol. 1301523

26. Mardiana S, John LB, Henderson MA, Slaney CY, von Scheidt B, Giuffrida L, et al. A Multifunctional Role for Adjuvant Anti-4-1bb Therapy in Augmenting Antitumor Response by Chimeric Antigen Receptor T Cells. Cancer Res (2017) 77(6):1296-309. doi: 10.1158/0008-5472.CAN-16-1831

27. Otano I, Azpilikueta A, Glez-Vaz J, Alvarez M, Medina-Echeverz J, CortesDominguez I, et al. CD137 (4-1BB) Costimulation of CD8(+) T Cells Is More Potent When Provided in Cis Than in Trans With Respect to CD3-TCR Stimulation. Nat Commun (2021) 12(1):7296. doi: 10.1038/s41467-02127613-w

28. Chmielewski M, Hombach A, Heuser C, Adams GP, Abken H. T Cell Activation by Antibody-Like Immunoreceptors: Increase in Affinity of the Single-Chain Fragment Domain Above Threshold Does Not Increase T Cell Activation Against Antigen-Positive Target Cells But Decreases Selectivity. J Immunol (2004) 173(12):7647-53. doi: 10.4049/jimmunol.173.12.7647

29. Liu X, Jiang S, Fang C, Yang S, Olalere D, Pequignot EC, et al. Affinity-Tuned ErbB2 or EGFR Chimeric Antigen Receptor T Cells Exhibit an Increased Therapeutic Index Against Tumors in Mice. Cancer Res (2015) 75(17):3596607. doi: 10.1158/0008-5472.CAN-15-0159

30. Caruso HG, Hurton LV, Najjar A, Rushworth D, Ang S, Olivares S, et al. Tuning Sensitivity of CAR to EGFR Density Limits Recognition of Normal Tissue While Maintaining Potent Antitumor Activity. Cancer Res (2015) 75 (17):3505-18. doi: 10.1158/0008-5472.CAN-15-0139

31. Schmid DA, Irving MB, Posevitz V, Hebeisen M, Posevitz-Fejfar A, Sarria JC, et al. Evidence for a TCR Affinity Threshold Delimiting Maximal CD8 T Cell Function. J Immunol (2010) 184(9):4936-46. doi: 10.4049/jimmunol.1000173

32. Thomas S, Xue SA, Bangham CR, Jakobsen BK, Morris EC, Stauss HJ. Human T Cells Expressing Affinity-Matured TCR Display Accelerated Responses But Fail to Recognize Low Density of MHC-Peptide Antigen. Blood (2011) 118 (2):319-29. doi: 10.1182/blood-2010-12-326736

33. Chervin AS, Stone JD, Soto CM, Engels B, Schreiber H, Roy EJ, et al. Design of T-Cell Receptor Libraries With Diverse Binding Properties to Examine Adoptive T-Cell Responses. Gene Ther (2013) 20(6):634-44. doi: 10.1038/ gt.2012.80 
34. Turatti F, Figini M, Balladore E, Alberti P, Casalini P, Marks JD, et al. Redirected Activity of Human Antitumor Chimeric Immune Receptors Is Governed by Antigen and Receptor Expression Levels and Affinity of Interaction. J Immunother (2007) 30(7):684-93. doi: 10.1097/CJI. 0b013e3180de5d90

35. Ghorashian S, Kramer AM, Onuoha S, Wright G, Bartram J, Richardson R, et al. Enhanced CAR T Cell Expansion and Prolonged Persistence in Pediatric Patients With ALL Treated With a Low-Affinity CD19 CAR. Nat Med (2019) 25(9):1408-14. doi: 10.1038/s41591-019-0549-5

36. Ma P, Ren P, Zhang C, Tang J, Yu Z, Zhu X, et al. Avidity-Based Selection of Tissue-Specific CAR-T Cells From a Combinatorial Cellular Library of CARs. Adv Sci (Weinh) (2021) 8(6):2003091. doi: 10.1002/advs. 202003091

37. Davenport AJ, Cross RS, Watson KA, Liao Y, Shi W, Prince HM, et al. Chimeric Antigen Receptor T Cells Form Nonclassical and Potent Immune Synapses Driving Rapid Cytotoxicity. Proc Natl Acad Sci USA (2018) 115: E2068-76. doi: 10.1073/pnas.1716266115

38. Park S, Shevlin E, Vedvyas Y, Zaman M, Park S, Hsu YS, et al. Micromolar Affinity CAR T Cells to ICAM-1 Achieves Rapid Tumor Elimination While Avoiding Systemic Toxicity. Sci Rep (2017) 7(1):14366. doi: 10.1038/s41598017-14749-3

39. Zhao Z, Condomines M, van der Stegen SJC, Perna F, Kloss CC, Gunset G, et al. Structural Design of Engineered Costimulation Determines Tumor Rejection Kinetics and Persistence of CAR T Cells. Cancer Cell (2015) 28 (4):415-28. doi: 10.1016/j.ccell.2015.09.004

40. Katsarou A, Sjostrand M, Naik J, Mansilla-Soto J, Kefala D, Kladis G, et al. Combining a CAR and a Chimeric Costimulatory Receptor Enhances T Cell Sensitivity to Low Antigen Density and Promotes Persistence. Sci Transl Med (2021) 13(623):eabh1962. doi: 10.1126/scitranslmed.abh1962
Author Disclaimer: The views expressed are those of the authors and not necessarily those of the NHS, the NIHR or the Department of Health.

Conflict of Interest: JM is CSO, scientific founder and shareholder of Leucid Bio, is a member of the scientific advisory board of Arovella Pharmaceutics Ltd and has undertaken consultancy work for Bristol-Meyers-Squibb, Juno, Celgene, Ellipses Pharma and Biotest. LH undertook a PhD studentship funded by Leucid Bio. DD and DL-Y have acted as consultants for Leucid Bio and DD has been employed by Leucid Bio following completion of this work. JM and LH are co-inventors on patent filings in relation to pCAR technology. $\mathrm{KD}$ and $\mathrm{AC}$ are employees of Lumicks.

The remaining authors declare that the research was conducted in the absence of any commercial or financial relationships that could be construed as a potential conflict of interest.

Publisher's Note: All claims expressed in this article are solely those of the authors and do not necessarily represent those of their affiliated organizations, or those of the publisher, the editors and the reviewers. Any product that may be evaluated in this article, or claim that may be made by its manufacturer, is not guaranteed or endorsed by the publisher.

Copyright (c) 2022 Halim, Das, Larcombe-Young, Ajina, Candelli, Benjamin, Dillon, Davies and Maher. This is an open-access article distributed under the terms of the Creative Commons Attribution License (CC BY). The use, distribution or reproduction in other forums is permitted, provided the original author(s) and the copyright owner(s) are credited and that the original publication in this journal is cited, in accordance with accepted academic practice. No use, distribution or reproduction is permitted which does not comply with these terms. 